\title{
Force sensorless fixed-time model-free impedance coordination control for uncertain underwater teleoperation systems
}

Yana Yang ( $\nabla$ yyn@ysu.edu.cn )

Yanshan University https://orcid.org/0000-0001-6006-8566

Yihang Chen

Yanshan University

Lu Gan

Yanshan University

Changchun Hua

Yanshan University

\section{Research Article}

Keywords: Underwater teleoperation systems, Impedance control, Time delay estimation, Fixed time control

Posted Date: February 28th, 2022

DOI: https://doi.org/10.21203/rs.3.rs-1383061/v1

License: (9) This work is licensed under a Creative Commons Attribution 4.0 International License. Read Full License 


\title{
Force sensorless fixed-time model-free impedance coordination control for uncertain underwater teleoperation systems
}

\author{
Yana Yang, Yihang Chen, Lu Gan, Changchun Hua
}

\begin{abstract}
New robust fixed-time model-free impedance controllers are proposed in this paper for coordination control of underwater teleoperation systems with system model uncertainties and unknown external underwater interference. Firstly, the transparency and the flexibility of the master-slave manipulators are improved without force sensor, respectively. A new terminal sliding mode force observer is designed to estimate force in fixed time by designing observer parameters. Moreover, time delay estimators are designed to compensate for the effect of model uncertainties and unknown external disturbances. Furthermore, a novel nonsingular integral terminal sliding mode (NITSM) surface and fixed-time controllers are proposed, so that master-slave coordination control can be obtained in fixed time with zero errors. The coordination performance and global stability of the underwater teleoperation systems are proved with the Lyapunov stability theory. Finally, simulation results highlight the feasibility of the proposed control scheme. The results show the super performance of the position and force coordination control for the underwater teleoperation systems.
\end{abstract}

Keywords: Underwater teleoperation systems; Impedance control; Time delay estimation; Fixed time control

\section{Introduction}

Underwater vehicles have been widely employed in many sea fields such as reconnaissance, undersea detection [1] and underwater surveillance [2]. With the development of submarine operations, underwater vehicles are required to finish more complex and sophisticated work such as exploration of underwater resources, oceanographic observation, underwater pipeline tracking [3], underwater structure maintenance [4], mine detection, and so on. However, it is difficult to complete complex work for underwater vehicles because of the dangerous underwater environment, uncertain operation objects and lacking object force feedback, which leads to over operation or miss operation problems. Therefore, the research of bilateral underwater teleoperation systems is a concerning direction to settle above problems [5]. The underwater teleoperation systems aims at teleoperation robotic manipulators mounted on vehicles in underwater environment.

Teleoperation systems play an important role in applications of telemedicine, accident rescue and space exploration [6]. Teleoperation systems extend the manipulator controlling ability to a remote environment and get the controlling situation of slave manipulator [7]. For the structure of the underwater teleoperation systems shown in Fig. 1, it contains a human operator located in the surface vessel or the manned submersibles, a master manipulator in the manned submersible, a communication channel connecting the manipulators, a slave manipulator mounted on the underwater vehicle, a remote underwater environment [8]. For the underwater teleoperation systems, the human operator applies force to the master manipulator, then the master information of force and position will be transformed to the slave manipulator by the communication channel after a time delay. The slave manipulator tracks the master manipulator's position and acts on the environment's object to

*The author is with the Institute of Electrical Engineering, Yanshan University, Qinhuangdao City, 066004, China. yyn@ysu.edu.cn 


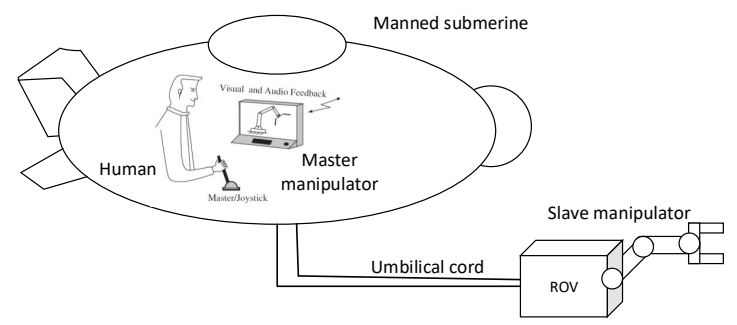

Figure 1: The structure of the bilateral underwater teleoperation systems

finish work. The environment force is feedback to the master manipulator, which makes the human operator feel the direct operation of the remote object.

For the underwater teleoperation systems with model uncertainties and external underwater disturbances, there are varieties of methods proposed to study position control and force control. For instance, reference [9] presents an adaptive controller based on neural network to achieve position tracking control. In [10], a new control method was proposed to estimate the external force. These control schemes achieve position or force tracking control, but the feedback force plus position relationship between the master and the slave is not reflected. So it is necessary to improve transparency and realize the force and position coordination control. The position and force synchronous control was achieved by adopting a proportional derivative (PD)-like controller with the effect of time delay in reference [11]. Mohammadi [12] employed a four-channel method to achieve force and position coordination control. In reference [13], a nonlinear-proportional plus nonlinear damping $(\mathrm{nP}+\mathrm{nD})$ controller was proposed to guarantee force and position tracking. The above methods have good position and force coordination control performance. However, the above references have two control objectives including position tracking control and force tracking control. It is necessary to achieve a unique control objective by establishing a balanced relationship between forces feedback and position. To solve the problem, impedance control is adopted to improve transparency and flexibility for underwater teleoperation systems. Two desired impedance models [14][15] are proposed for underwater teleoperation systems to provide the manipulators' desired trajectory. The impedance control models can regulate the reference trajectory tracked by the manipulators through changes of different contact forces, which improves the transparency and makes the teleoperation systems complete more precise work in applications. For the master impedance model, the desired trajectory is regulated by the forces error between the human operator force and the environment feedback force. And the master-slave force tracking is achieved by designing appropiate parameters. The master impedance model realizes force synchronization control and improves transparency. For the slave impedance model, the environment feedback force is input to realize master-slave position tracking control, which improves the flexibility of the manipulator. The impedance control method demands force information. A sliding mode force observer with adaptive gains was proposed to estimate force in reference [16], which can guarantee finite time coordination control. In reference [17], a new force observer was proposed to improve transparency and achieve force-position coordination control, but it needs correct model information. Compared with the references, a terminal sliding mode force observer is designed based on TDE without complex calculation rules. The operator applying force and environment feedback force are estimated by a designed force observer in fixed time.

It cannot be ignored that the master and slave manipulators are complex nonlinear systems with model uncertainties. Furthermore, the slave manipulator work in a dangerous environment with the interference of wind, wave and current. Then, a lot of control methods are promoted to deal with model uncertainties and external disturbances such as: proportional integral derivative (PID) control, adaptive control, fuzzy control, neural network control, backstepping control, sliding mode control, and so on [18]- [19]. In the presence of model uncertainties and external disturbances, general methods are not always effective and need exact model infor- 
mation. Therefore, the intelligent control methods can handle these problems and have a strong approximation ability. Several adaptive and neural network methods are proposed to estimate unknown parameters and model uncertainties. For the teleoperation systems with time-varying delays [18], an adaptive fuzzy controller was presented to eliminate the effect of model parameter uncertainties. However, the position tracking error between the manipulators and parameter estimation error is not zero but bounded. Reference [20] presents an adaptive controller for underwater vehicles to tackle external disturbances and model uncertainties, which has strong robustness and achieves better control performance. Reference [19] proposes an adaptive neural network controller to estimate uncertain parameters by adopting many times neural network learning and training. In reference [21], two adaptive controllers were designed based on neural network to achieve position tracking control. However, intelligent control methods are dependent on complex rules, which consist of a lot of designed parameters. In application, time delay estimation (TDE) can handle these problems, which is widely used in various systems such as robot manipulators [22], underwater vehicles [23], and quadrotor systems. TDE is independent of system models, which achieves model-free control and compensates for the effect of model uncertainties and external disturbances. For instance, TDE controllers in [24]- [25] were designed to achieve system state convergence without exact parameter information. In this paper, the combination of sliding mode surface with TDE has advantages such as good tracking accuracy, achieving mode-free coordination control, reducing settling time.

In recent years, approaches are widely proposed to achieve position and force coordination control for teleoperation systems. However, it has been noted that most of these methods achieve asymptotic/exponential convergence, which means the system state converges to the equilibrium point when the time approaches infinity [26]- [27]. Therefore, fast convergence performance can't be realized when the system achieves asymptotic/exponential convergence. To solve the problem of settling-time and improve control performance, finitetime control methods are employed for manipulators. Reference [28] proposes two adaptive finite-time control schemes based on neural networks and auxiliary compensation filters. In [29]- [30], integrating sliding mode surface and finite time control, new control approaches are proposed to achieve good control performance. The above schemes compensate for the effect of disturbance and time delay in finite time. But finite-time control is related to the initial value [31]- [32], and the settling time is related to the system initial value. Then fixed time control is proposed based on finite-time control, which is better than finite-time control and has the advantages of finite time. The settling time of fixed time control is decided by fixed-time controller parameters and is independent of the system initial value. It is of great significance to study this method and apply it to solve underwater teleoperation systems coordination control problems. Sliding mode control is an effective technique to guarantee tracking error convergence. Sliding mode control contains the reaching phase and sliding phase. The settling time is decided by control parameters and sliding mode parameters. In reference [33], a nonsingular terminal sliding mode was proposed to realize system states finite-time convergence and guarantee robustness of the system with unknown disturbance. Reference [34] proposed an adaptive nonsingular fixed-time sliding mode to achieve trajectory tracking under external disturbances. Compared with these control methods, a nonsingular integral terminal sliding mode surface is proposed to achieve fast convergence in this paper, which is better than the reference sliding mode because it can reduce the reaching phase. Combining the advantages of both fixed-time control and NITSM, a fixed time controller is designed to realize position tracking.

In this paper, a new robust fixed-time controller is proposed based on time delay estimation for synchronization control in underwater teleoperation systems. The combination of the fixed-time control method and time delay estimation technology realizes good tracking accuracy and reduces chattering. What's the most important influence of the control method is eliminating the effect of system model uncertainty and external disturbances without complex steps of measurement parameters, which is applied in practical production. The main contributions of this method are expressed as follows:

i) The transparency and the flexibility of the master-slave manipulators are improved by designing impedance 
control models. The impedance models balance the relationship between forces and reference position tracked by manipulators, which provide a unique impedance control target for underwater teleoperation systems rather than control force or position separately. The master-slave forces feedback and reference position tracking are reflected by impedance models. A novel terminal sliding mode force observer is designed to estimate the forces in fixed time.

ii) A novel time delay estimator is designed to compensate for the effect of model parameter uncertainty and unknown external disturbances without exact model information, which achieves zero-error model-free control.

iii) A fixed-time controller is designed based on the nonsingular integral terminal sliding surface to achieve that system state converges to equilibrium point in fixed time.

The rest of this paper is organized as follows. Section 2 presents some preliminary knowledge of the dynamics of underwater teleoperation systems. In section 3, a fixed-time controller and a force observer are proposed, and the stability analysis of the system is proven. The simulation results are shown in Section 4. Finally, Section 5 summarizes the obtained results.

Notation. $\mathbb{R}$ indicates the set of real nunmbers; $\mathbb{R}^{n}$ denotes the set of $n$ column vectors; $\mathbb{R}^{n \times m}$ represent the set of $n$ row $m$ column matrices, $\mathbb{R}_{+}$denotes positive real number. For a given matrix $X \in \mathbb{R}^{n \times n}, X^{-1}$ is the inverse matrix of $X$. For a vector $x=\left[x_{1}, \ldots, x_{n}\right]^{T}, \operatorname{sig}(x)^{\gamma}=\left[\left|x_{1}^{\gamma}\right| \operatorname{sign}\left(x_{1}\right), \ldots,\left|x_{n}^{\gamma}\right| \operatorname{sign}\left(x_{n}\right)\right]^{T}, \gamma$ is a positive constant. $\|x\|$ represents the 2-norm, $\|x\|_{\infty}$ denotes the infinite norm. $\lambda_{\min }(X)$ and $\lambda_{\max }(X)$ represent the minimum and maximum eigenvalues of matrix $X$, which only has real eigenvalues, respectively.

\section{Model and preliminaries}

Considering the nonlinear n-degree-of-freedom (n-DOF) model of underwater teleoperation systems with model uncertainties and external disturbances, which dynamics in joint space are given as:

$$
\begin{gathered}
M_{m}\left(q_{m}\right) \ddot{q}_{m}+C_{m}\left(q_{m}, \dot{q}_{m}\right) \dot{q}_{m}+G_{m}\left(q_{m}\right)=\tau_{m}-d_{m}\left(\dot{q}_{m}\right)-F_{h} \\
M_{s}\left(q_{s}\right) \ddot{q}_{s}+C_{s}\left(q_{s}, \dot{q}_{s}\right) \dot{q}_{s}+G_{s}\left(q_{s}\right)=\tau_{s}-d_{s}\left(\dot{q}_{s}\right)-d_{w}-F_{e}
\end{gathered}
$$

The subscript $i=m$ denotes the master manipulator controlled by humans and $i=s$ denotes the slave manipulator in underwater. $q_{i} \in \mathbb{R}^{n}$ is the vector of the joint position; $\dot{q}_{i} \in \mathbb{R}^{n}$ is the vector of joint velocity; $\ddot{q}_{i} \in \mathbb{R}^{n}$ is the vector of joint acceleration; $M_{i}\left(q_{i}\right) \in \mathbb{R}^{n \times n}$ denotes the positive definite inertia matrix; $C_{i}\left(q_{i}, \dot{q}_{i}\right) \in$ $\mathbb{R}^{n \times n}$ represents the matrix of centripetal and coriolis torques; $G_{i}\left(q_{i}\right) \in \mathbb{R}^{n}$ is the gravitational torque; $d_{i}\left(\dot{q}_{i}\right) \in$ $R^{n}$ is the viscous friction and the coulomb friction; $d_{w} \in \mathbb{R}^{n}$ is the uncertain underwater disturbance of slave manipulator; $F_{h} \in \mathbb{R}^{n}$ and $F_{e} \in \mathbb{R}^{n}$ are force torque caused by human operator applying to the master and environment feedback force induced by environment, respectively. The force and position information will be transmitted to improve coordination performance between the master and the slave manipulators. For the underwater teleoperation systems, the human operator applies force to the master manipulator, then the position information $q_{m}$ and force information $F_{h}$ are passed to the slave manipulator by the communication channel after $T_{m}(t)$ time interval. The slave manipulator transmits the information of force $F_{e}$ to the master manipulator after $T_{s}(t)$ time interval. The underwater teleoperation systems consider the effect of bounded time-varying delays of $T_{m}(t)$ and $T_{s}(t)$. Then the input and output signals of the communication channel are 
designed as

$$
\begin{aligned}
& q_{m}^{d}(t)=q_{m}\left(t-T_{m}(t)\right) \\
& \dot{q}_{m}^{d}(t)=\dot{q}_{m}\left(t-T_{m}(t)\right) \\
& F_{e}^{d}(t)=F_{e}\left(t-T_{s}(t)\right)
\end{aligned}
$$

It's difficult to design controllers to track two targets (force and position) for underwater teleoperation systems in previous work. According to a reference [35], the impedance models were employed to reflect the relationship between the reference position and manipulators' forces error. So the master and slave manipulators have the only control objective, which is impedance regulation. The impedance models reflect the master-slave force feedback as input and guarantee force tracking, which provide a reference position tracked by manipulators by designing the controller. The transparency is improved by force feedback and master-slave force tracking. The flexibility is improved by choosing appropriate sufficients according to different work objectives. The impedance models reflect the relationship between force and position, which provide the master-slave manipulators' reference trajectory $q_{\bmod }$ and $q_{\bmod }$. The desired impedance models of the master and slave manipulators are designed as follows:

$$
\begin{gathered}
m_{m} \ddot{q}_{\bmod _{m}}+c_{m} \dot{q}_{\bmod _{m}}=\hat{F}_{h}-k_{f} \hat{F}_{e}^{d} \\
m_{s} \ddot{\tilde{q}}_{\text {mod }_{s}}+c_{s} \dot{\tilde{q}}_{m_{\text {od }}}+k_{s} \tilde{q}_{m o d_{s}}=-\hat{F}_{e}
\end{gathered}
$$

where $q_{\bmod m}$ and $q_{\bmod }$ are the outputs of the desired impedance model, standing for the desired trajectory in joint space. $\tilde{q}_{\text {mods }_{s}}=q_{\bmod _{s}}-k_{p} q_{m}^{d}$ is the error of the slave reference impedance model's response concerning for the scaled master position. The master impedance model inputs are estimated force $\hat{F}_{h}$ and $\hat{F}_{e}^{d}$. The term $\hat{F}_{h}$ denotes the value of $F_{h}$ and $\hat{F}_{e}^{d}$ denotes the value of $F_{e}^{d}$ estimated by force observers. $m_{i}$ and $c_{i}$ represent the desired virtual mass and damping coefficients of the target impedance model respectively; $k_{s}$ denotes the stiffness matrix for the slave manipulator; $k_{f}$ is the force scaling factor; $k_{p}$ is the position scaling factor.

Remark 1. The proposed reference impedance models achieve the position and force coordination control by designing the parameters of the equation (4) and (5). For the master impedance control model, the estimated human operator force $\hat{F}_{h}$ is input of the master manipulator in controlling velocity and acceleration $\left(\dot{q}_{m o d_{m}}\right.$ and $\left.\ddot{q}_{\bmod _{m}}\right)$ at the begining. Considering the practical applications, the velocity and acceleration $\left(\dot{q}_{m o d_{m}}\right.$ and $\left.\ddot{q}_{m o d_{m}}\right)$ are small and the coefficient $\left(m_{m}\right.$ and $\left.c_{m}\right)$ are chosen sufficiently small, which cause the right of the equation (4) becomes small. Therefore, the environment feedback force is approximately equal to the human operator force $\left[\left(\hat{F}_{h}-k_{f} \hat{F}_{e}^{d}\right) \rightarrow 0\right]$. However, if the value $\left(\hat{F}_{h}\right.$ or $\left.\hat{F}_{e}^{d}\right)$ on the right of the equation of impedance model becomes large, which lead to large velocity and acceleration $\left(\dot{q}_{m o d_{m}}\right.$ and $\left.\ddot{q}_{m o d_{m}}\right)$. The transparency performance is improved and the reference position is adjusted by the human operator force. In the slave reference impedance model (5), the environment feedback force $\hat{F}_{e}$ is bounded. If the parameters $m_{s}, c_{s}$ and $k_{s}$ are chosen large values, the tracking error $\tilde{q}_{\text {mod }_{s}}$ is extremely small and the position tracking $\left(q_{\bmod _{s}} \rightarrow k_{p} q_{m}^{d}\right)$ is obtained.

Definition 1. (finite-time stable) [36]: For a nonlinear system

$$
\dot{x}=g(t, x), x(0)=x_{0}
$$

where $x \in \mathbb{R}^{n}$ denotes the system state, and $g \in \mathbb{R}_{+} \times \mathbb{R}^{n} \rightarrow \mathbb{R}^{n}$ is considered to be nonlinear and discontinous. The origin of the system is said to be globally finite-time stable if it is globally asymptotically stable and the solution $x\left(t, x_{0}\right)$ of system (6) reaches the equilibrium point at some finite time moment, $\forall t \geq T\left(x_{0}\right): x\left(t, x_{0}\right)=$ 0 , where $T: \mathbb{R}^{n} \rightarrow \mathbb{R}_{+} \cup\{0\}$ is called the settling time function.

Definition 2. (fixed-time stable) [37]: The origin of system (6) is said to be globally fixed-time stable if it is globally uniformly finite-time stable and the settling-time function $T$ is globally bounded, $\exists T_{\max } \in \mathbb{R}_{+}$such that $T\left(x_{0}\right) \leq T_{\max }, \forall x_{0} \in \mathbb{R}^{n}$. 
Lemma 1. [38] Considering a scalar system

$$
\dot{y}=-\alpha \operatorname{sig}(y)^{\gamma_{1}}-\beta \operatorname{sig}(y)^{\gamma_{2}}, y(0)=y_{0}
$$

where $\alpha>0, \beta>0, \gamma_{1}>1$, and $0<\gamma_{2}<1$. The system is globally fixed-time stable with the settling time $T$ bounded by

$$
T=\frac{1}{\alpha} \frac{1}{\gamma_{1}-1}+\frac{1}{\beta} \frac{1}{1-\gamma_{2}}, \forall y_{0} \in \mathbb{R}^{n}
$$

Lemma 2. [38] Let $a_{1}, a_{2}, \ldots, a_{n} \geq 0$, if $p>1$, then

$$
\left(a_{1}+a_{2}+\cdots+a_{n}\right)^{p} \leq \max \left(n^{p-1}, 1\right)\left(a_{1}^{p}+a_{2}^{p}+\cdots+a_{n}^{p}\right)
$$

\section{Controller design and stability analysis}

\subsection{Fixed-time controller design based TDE and stability analysis}

By introducing a positive diagonal gain matrix $\bar{M}_{i i}=\operatorname{diag}\left(\bar{M}_{i 11}, \bar{M}_{i 22}, \ldots, \bar{M}_{i n n}\right) \in \mathbb{R}^{n \times n}, i=m, s$, the force $F_{h}$ and $F_{e}$ are written as $F_{m h e}$ and $F_{\text {she }}$, system (1) and (2) can be expressed as follows:

$$
\begin{gathered}
\bar{M}_{m m} \ddot{q}_{m}+\left[M_{m}\left(q_{m}\right)-\bar{M}_{m m}\right] \ddot{q}_{m}+C_{m}\left(q_{m}, \dot{q}_{m}\right) \dot{q}_{m} \\
+G_{m}\left(q_{m}\right)+d_{m}\left(\dot{q}_{m}\right)+F_{m h e}=\tau_{m} \\
\bar{M}_{s s} \ddot{q}_{s}+\left[M_{s}\left(q_{s}\right)-\bar{M}_{s s}\right] \ddot{q}_{s}+C_{s}\left(q_{s}, \dot{q}_{s}\right) \dot{q}_{s} \\
+G_{s}\left(q_{s}\right)+d_{s}\left(\dot{q}_{s}\right)+d_{w}+F_{s h e}=\tau_{s}
\end{gathered}
$$

For the simplicity of (10) and (11), define

$$
\begin{gathered}
N_{m}=\left[M_{m}\left(q_{m}\right)-\bar{M}_{m m}\right] \ddot{q}_{m}+C_{m}\left(q_{m}, \dot{q}_{m}\right) \dot{q}_{m}+G_{m}\left(q_{m}\right)+d_{m}\left(\dot{q}_{m}\right) \\
N_{s}=\left[M_{s}\left(q_{s}\right)-\bar{M}_{s s}\right] \ddot{q}_{s}+C_{s}\left(q_{s}, \dot{q}_{s}\right) \dot{q}_{s}+G_{s}\left(q_{s}\right)+d_{s}\left(\dot{q}_{s}\right)+d_{w}
\end{gathered}
$$

Introducing (12) and (13) into (10) and (11), the equation is expressed as

$$
\bar{M}_{i i} \ddot{q}_{i}+N_{i}+F_{\text {ihe }}=\tau_{i}
$$

In this paper, TDE is used to estimate the model uncertainties and external disturbances. The advantage of TDE is to achieve model independent control without complex approximate rules. For TDE control rules, the term of model uncertainties and external disturbances $N_{i}$ is estimated by $\hat{N}_{i}$. When the sampling time $L$ is designed small enough, $\hat{N}_{i}$ can be estimated as $N_{i_{t-L}}$ by using TDE. The principle of TDE is using delayed measurement signals to estimate the lumped model uncertainties and unknown external disturbance. The sampling time $L$ is different from time delay. And the time delay between the master-slave manipulators has not an effect on the sampling time $L$. The method TDE aims at the independent master or slave system. In general, the results of TDE is expressed as

$$
\hat{N}_{i}=N_{i_{t-L}}=\tau_{i_{t-L}}-\bar{M}_{i i} \ddot{q}_{i_{t-L}}-\hat{F}_{i h e_{t-L}}
$$

where $\hat{F}_{i h e_{t-L}}$ is the estimated value of $F_{i h e}$ after time delay $L$. The bounded TDE error is designed as

$$
\varepsilon_{i}=\hat{N}_{i}-N_{i}
$$




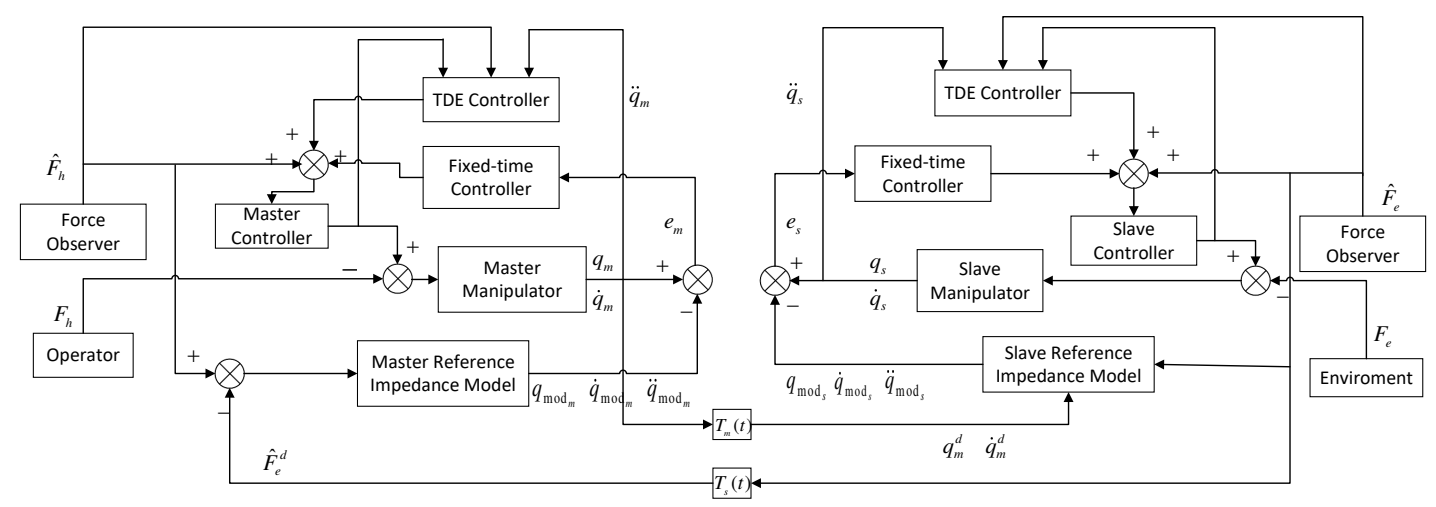

Figure 2: The control structure of underwater teleoperation systems.

Lemma 3. [39] It is derived that the TDE error $\varepsilon_{i}$ bounded and TDE error satisfying $\varepsilon_{i} \leq \varepsilon_{\text {imax }}$ ( [39][40], [23]). The upper bound of $\varepsilon_{\text {imax }}$ is defined as $\varepsilon_{\text {imax }} \geq\left\|\hat{N}_{i}-N_{i}\right\|_{\infty}$ with a designed constant gain matrix $\bar{M}_{i i}$ satisfying

$$
\left\|I-M^{-1}(q) \bar{M}_{i i}\right\|<1
$$

The positive definite symmetric inertial matrix satisfies the condition that $0<M^{-} \leq\|M(q)\| \leq M^{+}$. $M^{-}$and $M^{+}$are defined positive constants, and $\|M(q)\|$ is the euclidean norm of $M(q)$. The constant diagonal gain matrix $\bar{M}_{i i}=\operatorname{diag}\left\{\bar{M}_{i 11}, \bar{M}_{i 22}, \ldots, \bar{M}_{i n n}\right\}$ is designed to satisfies the equation of (17).

For the underwater teleoperation systems, the reference trajectory $q_{\text {mod }_{i}}$ is tracked by the manipulator $q_{i}$ obtained through the impedance model. In order to achieve the target, a novel NITSM is designed as

$$
s_{i}=\int_{0}^{t} \alpha_{i 2} \operatorname{sig}\left(\dot{e}_{i}\right)^{p_{i 2}}+\alpha_{i 1} \operatorname{sig}\left(e_{i}\right)^{p_{i 1}}+\beta_{i 2} \operatorname{sig}\left(\dot{e}_{i}\right)^{q_{i 2}}+\beta_{i 1} \operatorname{sig}\left(e_{i}\right)^{q_{i 1}} \mathrm{~d} \tau+\dot{e}_{i}
$$

where $e_{m}=q_{m}-q_{m o d_{m}}$ and $e_{s}=q_{s}-q_{\bmod _{s}}$ are manipulators position errors between the desired impedance models (4)-(5) and the actual joint position. In this equation, $\alpha_{i 1}, \alpha_{i 2}, \beta_{i 1}, \beta_{i 2}$ are positive constants, $p_{i 1}$, $p_{i 2}, q_{i 1}, q_{i 2}$ are designed positive constants, and $1<p_{i 2}<2, p_{i 1}=\frac{p_{i 2}}{2-p_{i 2}}, 0<q_{i 2}<1, q_{i 1}=\frac{q_{i 2}}{2-q_{i 2}}$, which guaranteeing the stability performance of the sliding mode surface.

Remark 2. In this paper, a new nonsingular integral terminal sliding mode (NITSM) surface is proposed, which avoids the singularity problem and reduces the settling time. When the system state is far from the equilibrium points, the terms $p_{i 2}, p_{i 1}$ make a difference to the system state convergence, producing a fast convergence rate and obtaining uniform convergence. When the system state approximates the equilibrium points, the effect of the terms $\beta_{i 1}, \beta_{i 2}$ is strong. Therefore, the system state converges to the equilibrium points in finite time. In addition, the advantage of the NITSM is reducing the reaching phase by choosing approximate initial values, which makes the system state on the sliding mode surface at the beginning.

The master and slave manipulators controllers are designed based on TDE in joint space, which is expressed as follows:

$$
\begin{aligned}
\tau_{i}= & -\varepsilon_{i \max } \operatorname{sign}\left(s_{i}\right)+\hat{N}_{i}+\hat{F}_{i h e}+\bar{M}_{i i}\left[\ddot{q}_{\text {mod }_{i}}-\alpha_{i 2} \operatorname{sig}\left(\dot{e}_{i}\right)^{p_{i 2}}\right. \\
& -\alpha_{i 1} \operatorname{sig}\left(e_{i}\right)^{p_{i 1}}-\beta_{i 2} \operatorname{sig}\left(\dot{e}_{i}\right)^{q_{i 2}}-\beta_{i 1} \operatorname{sig}\left(e_{i}\right)^{q_{i 1}} \\
& \left.-K_{i 1} \operatorname{sig}\left(s_{i}\right)^{\rho_{1}}-K_{i 2} \operatorname{sig}\left(s_{i}\right)^{\rho_{2}}\right]
\end{aligned}
$$


where $K_{m 1}, K_{m 2}, K_{s 1}, K_{s 2}$ are diagonal positive-definite constant matrices and $\rho_{1}, \rho_{2}$ are positive constants satisfying that $\rho_{1}>1,0<\rho_{2}<1$. The force torque $\hat{F}_{i h e}$ is estimated by a designed observer. The control structure of the underwater teleoperation systems is shown in Fig.2.

Theorem 1. Considering the underwater teleoperation systems (1) and (2) with estimated force $\hat{F}_{h}$ and estimated environment force $\hat{F}_{e}$, the fixed-time controller (19), the conclusion are drawn as follows. (i) If $s_{i}=0$ at the beginning, then $s_{i}=0$ will be hold. (ii) If $s_{i} \neq 0$, the system state will converges to $s_{i}=0$ in fixed time under the action of controller parameters. (iii) The synchronization error of the master and slave manipulators will converge to zero in fixed time. The exact reaching time is expressed as:

$$
T<\frac{1}{A} \frac{1}{\rho_{1}-1}+\frac{1}{B} \frac{1}{1-\rho_{2}}
$$

where $\bar{K}_{1}=\min \left(\lambda_{\min }\left(K_{m 1}\right), \lambda_{\min }\left(K_{s 1}\right)\right), \bar{K}_{2}=\min \left(\lambda_{\min }\left(K_{m 2}\right), \lambda_{\min }\left(K_{s 2}\right)\right) . A=\bar{K}_{1} n^{\frac{1-\rho_{1}}{2}}, B=\bar{K}_{2}$.

Proof: The impedance control models provide the desired trajectory tracked by the master-slave manipulators $\left(q_{i} \rightarrow q_{\text {mod }_{i}}\right)$. According to the slave impedance model (5), this shows the slave impedance reference trajectory track the master manipulator trajectory $\left(q_{\bmod _{s}} \rightarrow q_{m}^{d}\right)$. In order to prove the stability of designed controller, a positive definite Lyapunov function candidate is designed as

$$
V=\frac{1}{2} s_{m}^{T} s_{m}+\frac{1}{2} s_{s}^{T} s_{s}
$$

Differentiating the Lyapunov function candidate $V$, which is expressed as

$$
\begin{aligned}
\dot{V}= & s_{m}^{T} \dot{s}_{m}+s_{s}^{T} \dot{s}_{s} \\
= & s_{m}^{T}\left[\ddot{q}_{m}-\ddot{q}_{m o d_{m}}+\alpha_{m 2} \operatorname{sig}\left(\dot{e}_{m}\right)^{p_{m 2}}+\alpha_{m 1} \operatorname{sig}\left(e_{m}\right)^{p_{m 1}}\right. \\
& \left.+\beta_{m 2} \operatorname{sig}\left(\dot{e}_{m}\right)^{q_{m 2}}+\beta_{m 1} \operatorname{sig}\left(e_{m}\right)^{q_{m 1}}\right] \\
& +s_{s}^{T}\left[\ddot{q}_{s}-\ddot{q}_{m o d_{s}}+\alpha_{s 2} \operatorname{sig}\left(\dot{e}_{s}\right)^{p_{s 2}}+\alpha_{s 1} \operatorname{sig}\left(e_{s}\right)^{p_{s 1}}\right. \\
& \left.+\beta_{s 2} \operatorname{sig}\left(\dot{e}_{s}\right)^{q_{s 2}}+\beta_{s 1} \operatorname{sig}\left(e_{s}\right)^{q_{s 1}}\right] \\
= & s_{m}^{T}\left[\bar{M}_{m m}^{-1}\left(\hat{N}_{m}-N_{m}-\varepsilon_{m m a x} \operatorname{sign}\left(s_{m}\right)\right)-K_{m 1} \operatorname{sig}\left(s_{m}\right)^{\rho_{1}}-K_{m 2} \operatorname{sig}\left(s_{m}\right)^{\rho_{2}}\right] \\
& +s_{s}^{T}\left[\bar{M}_{s s}^{-1}\left(\hat{N}_{s}-N_{s}-\varepsilon_{s m a x} \operatorname{sign}\left(s_{s}\right)\right)-K_{s 1} \operatorname{sig}\left(s_{s}\right)^{\rho_{1}}-K_{s 2} \operatorname{sig}\left(s_{s}\right)^{\rho_{2}}\right] \\
= & s_{m}^{T}\left[\bar{M}_{m m}^{-1}\left(\varepsilon_{m}-\varepsilon_{m \max } \operatorname{sign}\left(s_{m}\right)\right)-K_{m 1} \operatorname{sig}\left(s_{m}\right)^{\rho_{1}}-K_{m 2} \operatorname{sig}\left(s_{m}\right)^{\rho_{2}}\right] \\
& +s_{s}^{T}\left[\bar{M}_{s s}^{-1}\left(\varepsilon_{s}-\varepsilon_{s m a x} \operatorname{sign}\left(s_{s}\right)\right)-K_{s 1} \operatorname{sig}\left(s_{s}\right)^{\rho_{1}}-K_{s 2} \operatorname{sig}\left(s_{s}\right)^{\rho_{2}}\right] \\
&
\end{aligned}
$$

With the difinition of $\varepsilon_{i}$, applying the term of $\varepsilon_{i \max } \geq\left\|\hat{N}_{i}-N_{i}\right\|_{\infty}$ and lemma 2, it concludes that

$$
\begin{aligned}
\dot{V} \leq & -s_{m}^{T} K_{m 1} \operatorname{sig}\left(s_{m}\right)^{\rho_{1}}-s_{m}^{T} K_{m 2} \operatorname{sig}\left(s_{m}\right)^{\rho_{2}}-s_{s}^{T} K_{s 1} \operatorname{sig}\left(s_{s}\right)^{\rho_{1}}-s_{s}^{T} K_{s 2} \operatorname{sig}\left(s_{s}\right)^{\rho_{2}} \\
\leq & -\lambda_{\min }\left(K_{m 1}\right) n^{\frac{1-\rho_{1}}{2}}\left(s_{m}^{T} s_{m}\right)^{\frac{\rho_{1}+1}{2}}-\lambda_{\min }\left(K_{m 2}\right)\left(s_{m}^{T} s_{m}\right)^{\frac{\rho_{2}+1}{2}} \\
& -\lambda_{\min }\left(K_{s 1}\right) n^{\frac{1-\rho_{1}}{2}}\left(s_{s}^{T} s_{s}\right)^{\frac{\rho_{1}+1}{2}}-\lambda_{\min }\left(K_{s 2}\right)\left(s_{s}^{T} s_{s}\right)^{\frac{\rho_{2}+1}{2}}
\end{aligned}
$$

Applying lemma 2, it concludes that

$$
\begin{aligned}
\dot{V} & \leq-\bar{K}_{1} n^{\frac{1-\rho_{1}}{2}}\left(s_{m}^{T} s_{m}+s_{s}^{T} s_{s}\right)^{\frac{\rho_{1}+1}{2}}-\bar{K}_{2}\left(s_{m}^{T} s_{m}+s_{s}^{T} s_{s}\right)^{\frac{\rho_{2}+1}{2}} \\
& =-\bar{K}_{1} n^{\frac{1-\rho_{1}}{2}}(2 V)^{\frac{\rho_{1}+1}{2}}-\bar{K}_{2}(2 V)^{\frac{\rho_{2}+1}{2}}
\end{aligned}
$$

Then, define $y=\sqrt{2 V}$, we have

$$
\dot{y} \leq-\bar{K}_{1} n^{\frac{1-\rho_{1}}{2}} y^{\rho_{1}}-\bar{K}_{2} y^{\rho_{2}}
$$


On the basis of lemma 1, the system state converges to the equilibrium point in fixed time, the settling time is shown as the equation of (20). This completes the proof. Then the sliding mode surface converges to zero $\left(s_{i} \rightarrow 0\right)$ in fixed time, the position tracking error $e_{i} \rightarrow 0$ and $\dot{e}_{i} \rightarrow 0$, namely $q_{i} \rightarrow q_{\text {mod }_{i}}$. According to the impedance model, $q_{m} \rightarrow q_{\text {mod }_{m}}, q_{s} \rightarrow q_{\text {mod }_{s}}$ and $q_{\text {mod }_{s}} \rightarrow q_{m}^{d}$, which means $q_{s} \rightarrow q_{m}^{d}\left(q_{s} \rightarrow q_{m}\left(t-T_{m}(t)\right)\right)$. General control methods consider two control objectives including force and position tracking control with time dealy together. The corresponding stability is proved containing force and position control. In this paper, force tracking and position tracking are achieved by impedance control and NITSM control seperately. And the force tracking stability analysis has been shown above by using impedance. The position tracking stability analysis is achieved by using Lyapunov function. The position and force coordination control are realized in fixed time by adopting several techniques.

\subsection{Force observer design}

For the bilateral underwater teleoperation systems, there is a situation that the value of $F_{\text {ihe }}$ is unknown in practical application when the force sensor is insensitive or damaged in a dangerous underwater environment. Then the force $F_{i h e}$ in the equation of (10) and (11) are unknown. In order to obtain the value of $F_{\text {ihe }}$, a force observer is designed to estimate the value of force in fixed time. From the equation of (14), we get

$$
\ddot{q}_{i}=\bar{M}_{i i}^{-1}\left[\tau_{i}-N_{i}-F_{i h e}\right]
$$

Assumption 1. The first derivatives of the force exists as $\left|\dot{F}_{\text {ihe }}\right|<L_{1}$, the upper bound $L_{1}$ is a positive constant.

In order to design a force observer, an auxiliary variable is introduced as

$$
\epsilon_{i}=\dot{q}_{i}-z_{i}
$$

where the variable $z_{i}$ is designed as follows:

$$
\dot{z}_{i}=\bar{M}_{i i}^{-1}\left[\tau_{i}-\hat{N}_{i}-v_{z}\right]+\delta_{i} \operatorname{sig}\left(\epsilon_{i}\right)^{\gamma_{i 1}}+\theta_{i} \operatorname{sig}\left(\epsilon_{i}\right)^{\gamma_{i 2}}
$$

where $\delta_{i}$ and $\theta_{i}$ are positive constants, satisfing $\gamma_{i 1}>1,0<\gamma_{i 2}<1$. In the following theorem, $v_{z}$ is designed such that the variable $\epsilon_{i}$ and force estimation error converges to zero in fixed time.

Theorem 2. Considering a terminal sliding mode designed as following:

$$
\sigma_{i}=\dot{\epsilon}_{i}+\delta_{i} \operatorname{sig}\left(\epsilon_{i}\right)^{\gamma_{i 1}}+\theta_{i} \operatorname{sig}\left(\epsilon_{i}\right)^{\gamma_{i 2}}
$$

The control law of $v_{z}$ is designed as:

$$
\dot{v}_{z}=-\left(k_{a} \operatorname{sign}\left(\sigma_{i}\right)+\eta_{a} \operatorname{sig}\left(\sigma_{i}\right)^{\rho_{3}}+\eta_{b} \operatorname{sig}\left(\sigma_{i}\right)^{\rho_{4}}\right), v_{z}(0)=0
$$

where $\eta_{a}, \eta_{b}$ are positive constants, $0<\rho_{4}<1$ and $\rho_{3}>1$. If the condition satisfying $k_{a}>L_{1}$, then $\epsilon_{i}$ converges to zero in fixed time. Then the force $F_{i h e}$ can be estimated in fixed time, which is expressed as:

$$
\hat{F}_{\text {ihe }}=v_{z}
$$

Proof: The derivatives of $\epsilon_{i}$ is

$$
\dot{\epsilon}_{i}=\ddot{q}_{i}-\dot{z}=\bar{M}_{i i}^{-1}\left[v_{z}-F_{i h e}\right]-\delta_{i} \operatorname{sig}\left(\epsilon_{i}\right)^{\gamma_{i 1}}-\theta_{i} \operatorname{sig}\left(\epsilon_{i}\right)^{\gamma_{i 2}}
$$


For the equation of (32), the TDE estimation error $N_{i}-\hat{N}_{i}$ is ignored because the estimation error is sufficiently small for the value of force $F_{i h e}$. Then the force observer $v_{z}$ is designed to estimate the value of $F_{i h e}$ absolutely. By substituting the equation (32) into (29), the equation is expressed as

$$
\sigma_{i}=\bar{M}_{i i}^{-1}\left[v_{z}-F_{i h e}\right]
$$

Now consider the Lyapunov function candidate $V=\frac{1}{2} \sigma_{i}^{T} \bar{M}_{i i} \sigma_{i}$. By derivating the Lyapunov function candidate, we obtain

$$
\begin{aligned}
\dot{V} & =\sigma_{i}^{T} \bar{M}_{i i} \dot{\sigma}_{i} \\
& =\sigma_{i}^{T} \bar{M}_{i i} \bar{M}_{i i}^{-1}\left[\dot{v}_{z}-\dot{F}_{i h e}\right] \\
& =\sigma_{i}^{T}\left[-\left(k_{a} \operatorname{sign}\left(\sigma_{i}\right)+\eta_{a} \operatorname{sig}\left(\sigma_{i}\right)^{\rho_{3}}+\eta_{b} \operatorname{sig}\left(\sigma_{i}\right)^{\rho_{4}}\right)-\dot{F}_{i h e}\right] \\
& =-k_{a}\left|\sigma_{i}\right|-\sigma_{i}^{T} \dot{F}_{i h e}+\sigma_{i}^{T}\left[-\eta_{a} \operatorname{sig}\left(\sigma_{i}\right)^{\rho_{3}}-\eta_{b} \operatorname{sig}\left(\sigma_{i}\right)^{\rho_{4}}\right] \\
& \leq-k_{a}\left|\sigma_{i}\right|+\left|\sigma_{i}\right|\left|\dot{F}_{i h e}\right|-\sigma_{i}^{T} \eta_{a} \operatorname{sig}\left(\sigma_{i}\right)^{\rho_{3}}-\sigma_{i}^{T} \eta_{b} \operatorname{sig}\left(\sigma_{i}\right)^{\rho_{4}}
\end{aligned}
$$

Since $\eta_{a}>0, \eta_{b}>0$ and $k_{a}>L_{1}$, we obtain

$$
\begin{aligned}
\dot{V}= & \left|\sigma_{i}\right|\left(\left|\dot{F}_{i h e}\right|-k_{a}\right)-\frac{\lambda_{\min }\left(\eta_{a}\right)}{\lambda_{\max }\left(\bar{M}_{i i}\right)^{\frac{1+\rho_{3}}{2}}} n^{\frac{1-\rho_{3}}{2}}\left(\sigma_{i}^{T} \bar{M}_{i i} \sigma_{i}\right)^{\frac{1+\rho_{3}}{2}} \\
& -\frac{\lambda_{\min }\left(\eta_{b}\right)}{\lambda_{\max }\left(\bar{M}_{i i}\right)^{\frac{1+\rho_{4}}{2}}}\left(\sigma_{i}^{T} \bar{M}_{i i} \sigma_{i}\right)^{\frac{1+\rho_{4}}{2}} \\
\leq & -\frac{\lambda_{\min }\left(\eta_{a}\right)}{\lambda_{\max }\left(\bar{M}_{i i}\right)^{\frac{1+\rho_{3}}{2}}} n^{\frac{1-\rho_{3}}{2}}(2 V)^{\frac{1+\rho_{3}}{2}}-\frac{\lambda_{\min }\left(\eta_{b}\right)}{\lambda_{\max }\left(\bar{M}_{i i}\right)^{\frac{1+\rho_{4}}{2}}}(2 V)^{\frac{1+\rho_{4}}{2}}
\end{aligned}
$$

where $n=2$. Then define $Y=\sqrt{2 V}$, it conclude that

$$
\dot{Y} \leq-\frac{\lambda_{\min }\left(\eta_{a}\right)}{\lambda_{\max }\left(\bar{M}_{i i}\right)^{\frac{1+\rho_{3}}{2}}} n^{\frac{1-\rho_{3}}{2}} Y^{\rho_{3}}-\frac{\lambda_{\min }\left(\eta_{b}\right)}{\lambda_{\max }\left(\bar{M}_{i i}\right)^{\frac{1+\rho_{4}}{2}}} Y^{\rho_{4}}
$$

According to lemma 1, the system state reaches the equilibrium point in fixed time. When $\sigma_{i}=0$, we get

$$
\dot{\epsilon}_{i}=-\delta_{i} \operatorname{sig}\left(\epsilon_{i}\right)^{\gamma_{i 1}}-\theta_{i} \operatorname{sig}\left(\epsilon_{i}\right)^{\gamma_{i 2}}
$$

Considering lemma 1, the equation of (37) is fixed time stable. The force observer is proposed to estimate the force $F_{i h e}$, the estimation error is

$$
e_{f}=v_{z}-F_{i h e}
$$

From the equation of (31) and (33), causing the result $e_{f}=0$, the force observer estimates the force $F_{i h e}$ in fixed time accurately.

\section{Simulation}

In this section, the super performance of the proposed control method and control results are shown by simulation. Two degrees of freedom (2-DOF) manipulators are chosen in simulation, one denotes the master manipulator and the other denotes the slave manipulator. The simulation results are carried out on the MATLAB platform based on the impedance models and dynamics models. 


\subsection{Parameters selection}

The master and slave manipulators parameters values are designed as $m_{1}=1.5 \mathrm{~kg}, m_{2}=1.5 \mathrm{~kg}, l_{1}=1.5 \mathrm{~m}$, $l_{2}=1.5 \mathrm{~m}$. The inertia matrix $M_{i}\left(q_{i}\right)$ in Eq. (1) can be expressed as $M_{i}\left(q_{i}\right)=\left[M_{11}\left(q_{i}\right) M_{12}\left(q_{i}\right) ; M_{21}\left(q_{i}\right) M_{22}\left(q_{i}\right)\right]$, the element of $M_{i}\left(q_{i}\right)$ is designed as

$$
\begin{aligned}
& M_{11}\left(q_{i}\right)=2 m_{2} l_{1} l_{2} \cos \left(q_{2}\right)+m_{2} l_{2}^{2}+\left(m_{1}+m_{2}\right) l_{1}^{2} \\
& M_{12}\left(q_{i}\right)=m_{2} l_{1} l_{2} \cos \left(q_{2}\right)+m_{2} l_{2}^{2} \\
& M_{21}\left(q_{i}\right)=m_{2} l_{1} l_{2} \cos \left(q_{2}\right)+m_{2} l_{2}^{2} \\
& M_{22}\left(q_{i}\right)=m_{2} l_{2}^{2}
\end{aligned}
$$

The coriolis and centripetal matrix $C_{i}\left(q_{i}, \dot{q}_{i}\right)$ can be expressed as $C_{i}\left(q_{i}, \dot{q}_{i}\right)=\left[C_{11}\left(q_{i}, \dot{q}_{i}\right) C_{12}\left(q_{i}, \dot{q}_{i}\right) ; C_{21}\left(q_{i}, \dot{q}_{i}\right)\right.$ $\left.C_{22}\left(q_{i}, \dot{q}_{i}\right)\right]$, the element of $C_{i}\left(q_{i}, \dot{q}_{i}\right)$ is designed as

$$
\begin{aligned}
& C_{11}\left(q_{i}\right)=-m_{2} l_{1} l_{2} \sin \left(q_{2}\right) \dot{q}_{2} \\
& C_{12}\left(q_{i}\right)=-m_{2} l_{1} l_{2} \sin \left(q_{2}\right)\left(\dot{q}_{1}+\dot{q}_{2}\right) \\
& C_{21}\left(q_{i}\right)=m_{2} l_{1} l_{2} \sin \left(q_{2}\right) \dot{q}_{2} \\
& C_{22}\left(q_{i}\right)=0
\end{aligned}
$$

The gravity torques vector $g_{i}\left(q_{i}\right)$ is expressed as $g_{i}\left(q_{i}\right)=\left[g_{1}\left(q_{i 1}\right) ; g_{2}\left(q_{i 2}\right)\right]$, the element of $g_{i}\left(q_{i}\right)$ is designed as

$$
\begin{aligned}
& g_{1}\left(q_{i 1}\right)=\left(m_{1}+m_{2}\right) l_{1} g \cos \left(q_{2}\right)+m_{2} l_{2} g \cos \left(q_{1}+q_{2}\right) \\
& g_{2}\left(q_{i 2}\right)=m_{2} l_{2} g \cos \left(q_{1}+q_{2}\right)
\end{aligned}
$$

where $g=9.81 \mathrm{~N} / \mathrm{s}^{2}$, denotes the gravity acceleration.

From the dynamics of the master-slave manipulators, the master manipulator has the viscous friction and the coulomb friction torque because of the effect of uncertainty disturbance. And the slave manipulator is influenced by flow and unknown disturbance. The term of the friction and the coulomb friction are expressed as

$$
\begin{gathered}
d_{m}\left(\dot{q}_{m}\right)=\left[\begin{array}{c}
d_{m 1} \\
d_{m 2}
\end{array}\right]=\left[\begin{array}{c}
10 \dot{q}_{m 1}+5 \operatorname{sign}\left(\dot{q}_{m 1}\right) \\
10 \dot{q}_{m 2}+5 \operatorname{sign}\left(\dot{q}_{m 2}\right)
\end{array}\right] \\
d_{s}\left(\dot{q}_{s}\right)=\left[\begin{array}{c}
d_{s 1} \\
d_{s 2}
\end{array}\right]=\left[\begin{array}{l}
20 \dot{q}_{s 1}+10 \operatorname{sign}\left(\dot{q}_{s 1}\right) \\
20 \dot{q}_{s 2}+10 \operatorname{sign}\left(\dot{q}_{s 2}\right)
\end{array}\right]
\end{gathered}
$$

The term of flow disturbance and unknown underwater disturbances is designed as

$$
d_{w}=\left[\begin{array}{l}
d_{w 1} \\
d_{w 2}
\end{array}\right]=\left[\begin{array}{l}
0.6 \sin (t) \\
0.6 \sin (t)
\end{array}\right]
$$

For the underwater teleoperation systems, environment feedback force $F_{e}$ is related to the contact position when the slave manipulator acts on the working target, the environment feedback force is defined as 


$$
F_{e}=\left\{\begin{aligned}
k_{e}\left(q_{s}-q_{e}\right), & q_{s} \geq q_{e} \\
0, & q_{s}<q_{e}
\end{aligned}\right.
$$

where $q_{e}$ denotes the contact joint position between the slave manipulator and the object in the underwater environment. $k_{e}$ represents stiffness coefficient. The environment feedback force is zero when the slave manipulator works in free state, and it will produce contact force when the joint position $q_{s}>q_{e}$. The parameters in this situation are designed as $q_{e}=0.5, k_{e}=1.5$. The human operator force $F_{h}$ is defined as

$$
F_{h}=\left[\begin{array}{l}
F_{h 1} \\
F_{h 2}
\end{array}\right]=\left[\begin{array}{l}
20 \sin (0.1 t) \\
20 \sin (0.1 t)
\end{array}\right]
$$

In particular, the situation of free motion of the master-slave manipulators should be considered. The slave manipulator's position tracks the master manipulator's position when the forces are zero in a period of time. In this case, the human operator force and the environment feedback force are rewritten nonlinear to shown the system state in free motion.

According to the equation of (4) and (5), the impedance model provides the desired trajectory. The values of $m_{m}$ and $c_{m}$ are chosen sufficiently small. At the moment, the values of $m_{s}, c_{s}$ and $k_{s}$ are chosen enough large. Then the coefficient of the impedance model is given by:

$$
\begin{aligned}
m_{m} & =0.1 \quad c_{m}=0.3 \\
m_{s} & =1 \quad c_{s}=20.5 \quad k_{s}=100 \\
k_{p} & =1 \quad k_{f}=1
\end{aligned}
$$

For the simulation, The time-varying delay between the master and slave are designed as $T_{m}(t)=0.1+0.1 \sin (t)$ and $T_{s}(t)=0.1+0.2 \sin (t)$. The initial value of the master manipulator is designed as $q_{m}(0)=[5 ; 8], \dot{q}_{m}=[0 ; 0]$, the initial value of the desired master manipulator trajectory from the impedance model is $q_{\bmod _{m}}(0)=[0 ; 0]$, $\dot{q}_{\text {mod }_{m}}(0)=[0 ; 0]$. The initial value of the slave manipulator is designed as $q_{s}(0)=[5 ; 8], \dot{q}_{s}=[0 ; 0]$, the initial value of the desired slave manipulator trajectory from the impedance model is $q_{\text {mod }_{s}}(0)=[2 ; 5], \dot{q}_{\text {mod }_{s}}(0)=[0 ; 0]$.

TDE is used to eliminate the effect of model uncertainty and external disturbance. Time delay estimation realizes zero error model-free coordination control. According to lemma 3, the gain matrix satisfies that $\left\|I-M^{-1}(q) \bar{M}_{i i}\right\|<1$, the value of the inertia matrix is calculated based on the manipulator parameters. After calculation, the gain matrix is designed as $M_{i i}=\operatorname{diag}[0.789,0.611]$ satisfying the condition. It is difficult to get the value of joint acceleration $\ddot{q}$ with noise, which can be estimated by the joint position. The velocity $\dot{q}_{i}$ is calculated as $\dot{q}_{i}=\left(q_{i}-q_{i_{t-L}}\right) / L$ and acceleration is calculated as $\ddot{q}_{i_{t-L}}=\left(q_{i}-2 q_{i_{t-L}}+q_{i_{t-2 L}}\right) / L^{2}$ by the numerical differentiation, $L=0.001$ is sampling time.

For the NITSM, the parameters are chosen as $\alpha_{i 1}=1, \alpha_{i 2}=1, \beta_{i 1}=2, \beta_{i 2}=2, p_{i 2}=7 / 5, p_{i 1}=7 / 3$, $q_{i 2}=7 / 9, q_{i 1}=7 / 11$. The fixed-time controller parameter is designed as $k_{i 1}=\operatorname{diag}[10,10], k_{i 2}=\operatorname{diag}[2,2]$, $\rho_{1}=3, \rho_{2}=9 / 11$, the upper bound of estimation error is given as $\varepsilon_{i m a x}=0.6 \operatorname{sign}\left(s_{i}\right)$.

In order to explain the performance of fast convergence, a linear sliding mode surface, a nonsingular fast terminal sliding mode surface, and a simple nonsingular integral terminal sliding mode surface are proposed to compare with the designed NITSM in this paper. The linear sliding mode is designed as $s=\dot{e}+c e$ with $e=q-q_{d}, \dot{e}=\dot{q}-\dot{q}_{d}$, where $c=2, q$ is actual position and $q_{d}$ is the reference position. The nonsingular terminal sliding mode surface is designed as $s=e+\beta \operatorname{sig}(\dot{e})^{\gamma}$, where $\beta=2, \gamma=1.5$. The simple integral terminal sliding mode surface is designed as $s=\int_{0}^{t} \alpha_{2} \operatorname{sig}(\dot{e})^{p_{2}}+\beta_{2} \operatorname{sig}(e)^{q_{2}} \mathrm{~d} \tau+\dot{e}$. A second-order system is employed to verify 


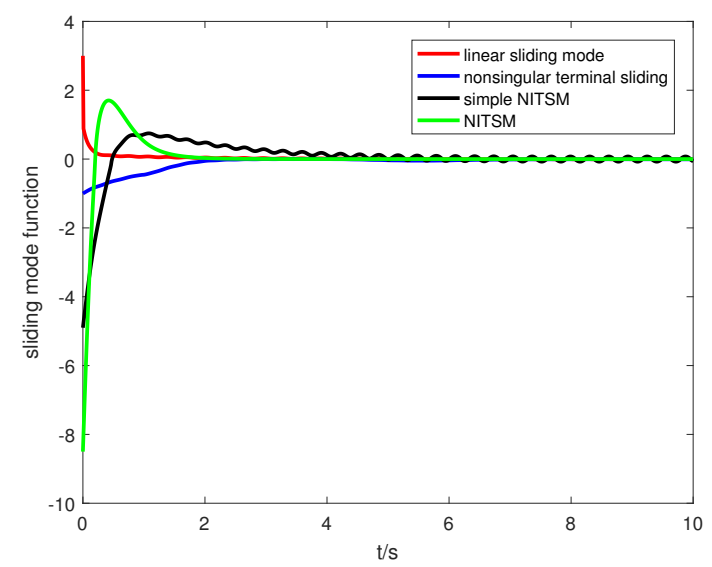

Figure 3: The values of sliding mode function

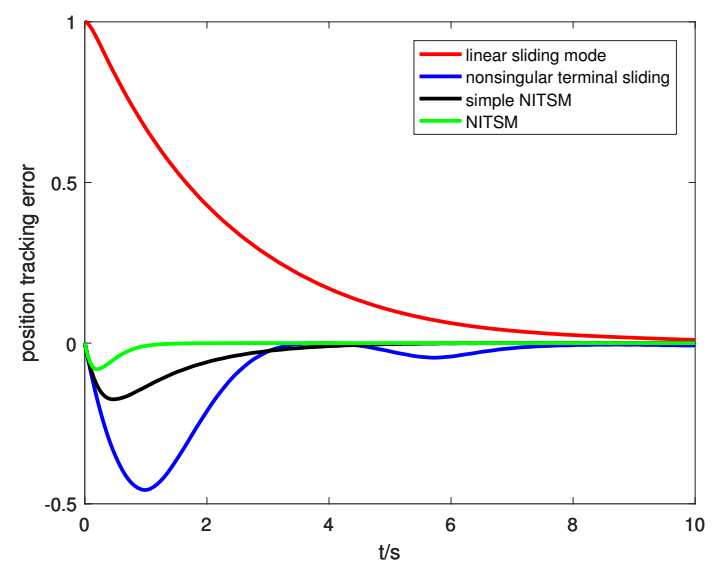

Figure 4: The values of position tracking error

convergence performance of different sliding mode surfaces. The settling time is compared by applying the above proposed sliding mode.

For the proposed terminal sliding mode force observer, the parameters are designed as $\delta_{i}=2, \theta_{i}=2, \gamma_{i 1}=3$, $\gamma_{i 2}=1 / 2, \rho_{3}=7 / 5, \rho_{4}=7 / 11, \eta_{a}=1, \eta_{b}=1.5, k_{a}=[2.10 ; 02.1]$.

\subsection{Simulation results}

In the simulation, according to the impedance models (4) and (5), the human operator force and remote environment feedback force are designed as above. What's more, the situation of free motion has been taken into consideration. The simulation time is set as 30s, the simulation results are shown in Fig.3-Fig.19.

Fig.3-5 showed the comparison results of different sliding mode surfaces. Fig.3 shows the value of different sliding mode surface. We can conclude that the designed NITSM has a short settling time better than nonsingular terminal sliding and simple NITSM. And the other sliding mode has chattering except NITSM in this paper. The designed NITSM has the best control performance. Fig.4-5 show the position tracking error and velocity tracking error. It is obvious that the proposed NITSM has good performance in settling time and is more stable than other sliding mode surfaces. By comparing these sliding mode surfaces, the proposed NITSM in this paper has good convergence performance. 


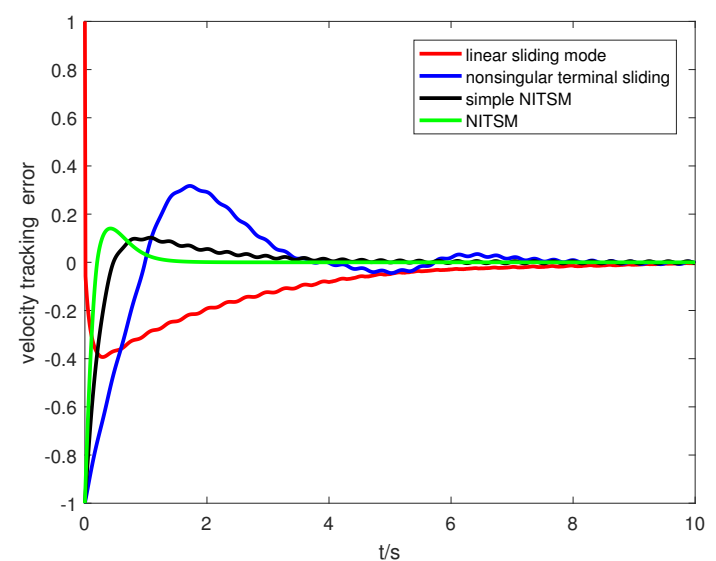

Figure 5: The values of velocity tracking error
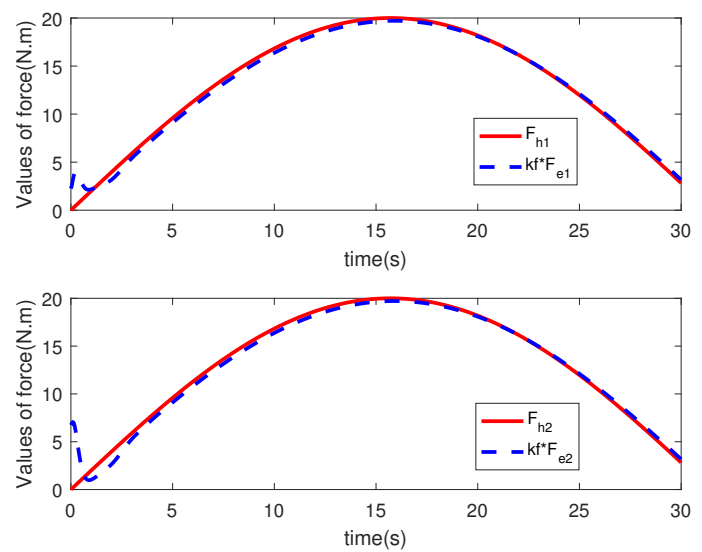

Figure 6: The values of human operator force and environment feedback force

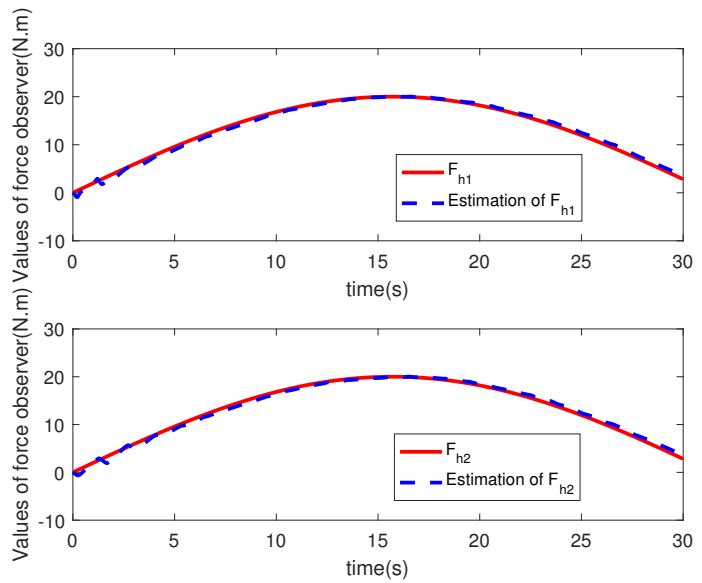

Figure 7: The values of human operator force and estimated force 


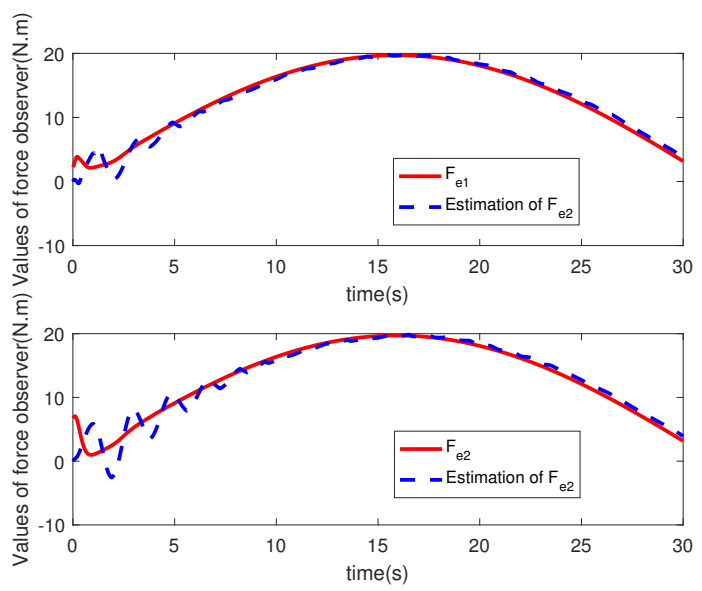

Figure 8: The values of environment feedback force and estimated force
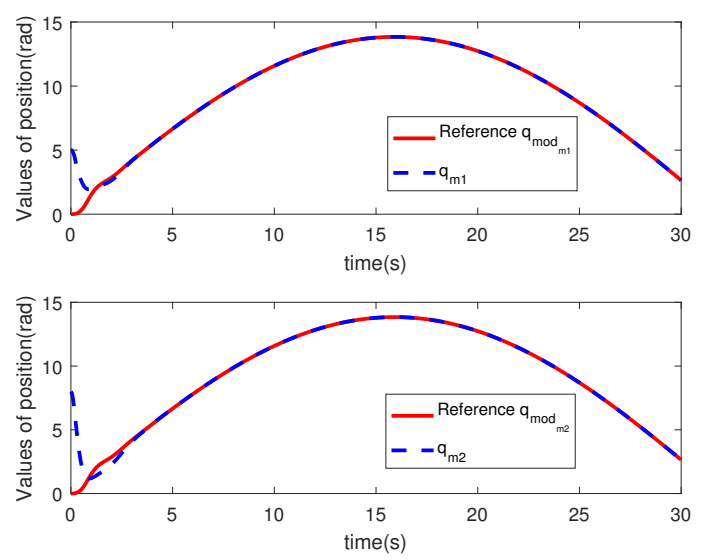

Figure 9: The values of reference impedance position and actual master position
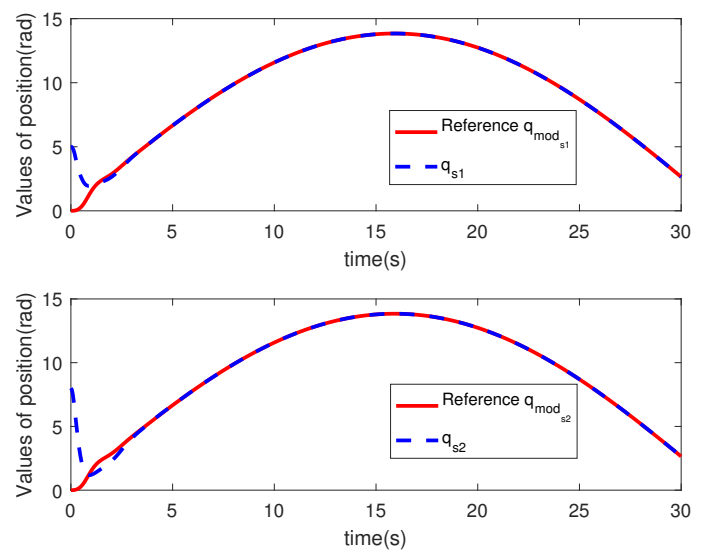

Figure 10: The values of reference impedance position and actual slave position 

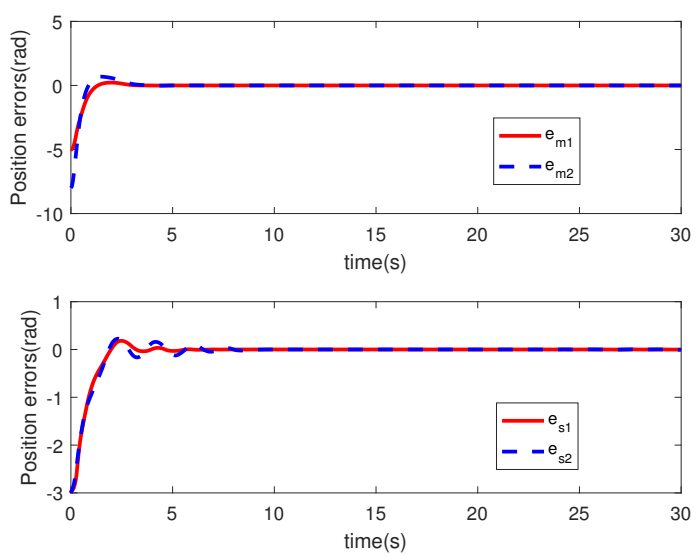

Figure 11: The values of position errors between desired impedance model and manipulators
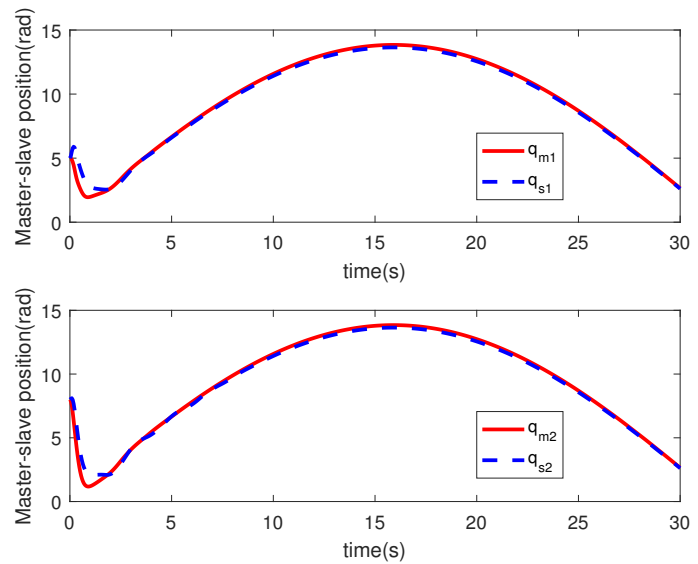

Figure 12: The values of position for master-slave manipulators
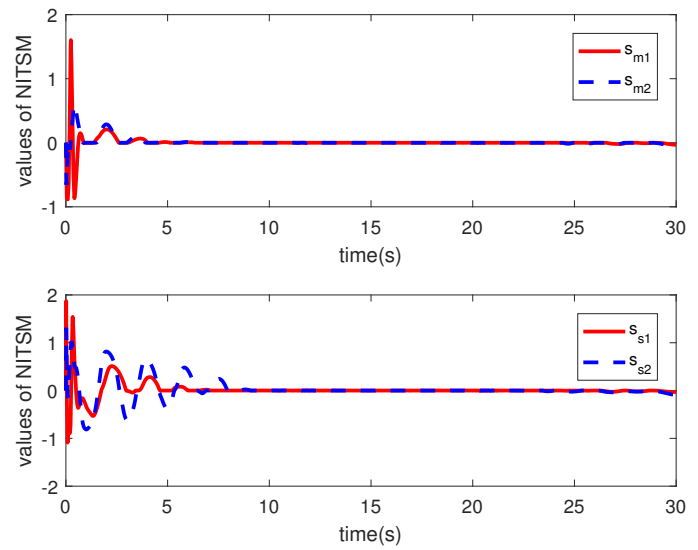

Figure 13: The values of NITSM for master-slave manipulators 

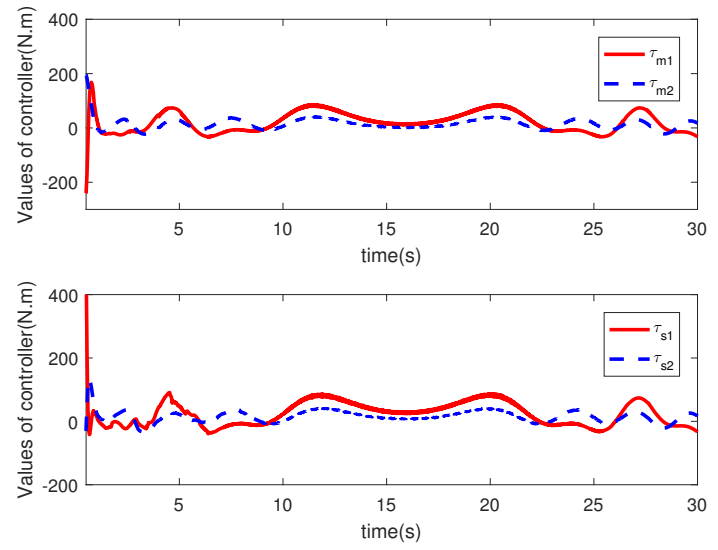

Figure 14: The values of controllers for master-slave manipulators
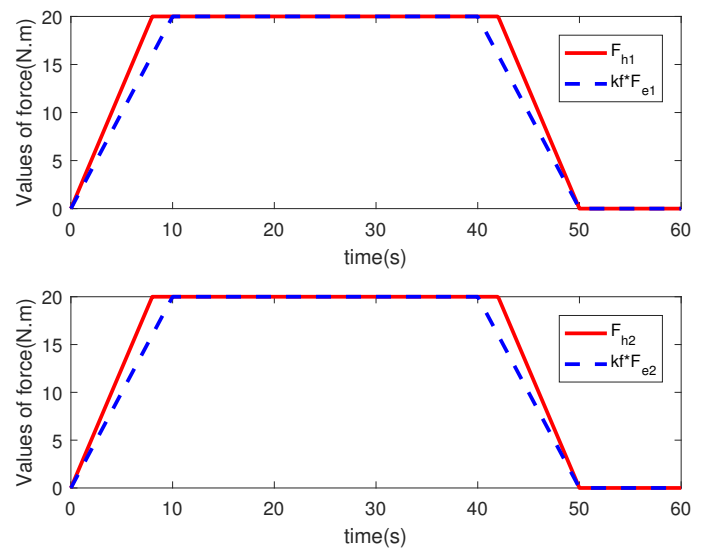

Figure 15: The values of human operator force and environment feedbcak force in free motion

In Fig.6, it shows the values of human operator force and environment feedback force. In Fig.7-8, it can be concluded that the designed fixed-time force observer estimates the force accurately. Fig.9-10 shows the manipulators' desired position trajectory $q_{\text {mod }_{i}}$ from the impedance control model and the actual position trajectory $q_{i}$. From the simulation results of the manipulators' position trajectory, we get that the master and slave manipulators can track the desired position trajectory in fixed time. The position synchronization errors between the master-slave manipulators' position and desired position are shown obviously in Fig.11, we can see that the synchronization error $e_{i}$ converges to zero in fixed time under the action of the controller. Fig.12 shows the actual position for master-slave manipulators, which gives a reflection that the master and slave trajectory is equal and the position error between the slave-master manipulators converges to zero in fixed time. The values of the NITSM surface for the manipulators are shown in Fig.13. From Fig.13, the system state converges $s_{i}=0$ in fixed time, which is affected by the controller parameter. The values of controllers are shown in Fig.14.

Considering the situation of free motion for underwater teleoperation systems, the simulation results have been shown in Fig.15-Fig.19. In free motion, the forces are designed nonlinear to explain accuracy force tracking control. The simulation time is designd as $60 \mathrm{~s}$. From Fig.15, the values of force change are showd. The manipulators' free motion is from $t=50 \mathrm{~s}$ to $t=60 \mathrm{~s}$ when master-slave forces are zero. According to Fig.16-17, the manipulators joint position $q_{i}$ can track the reference trajectory $q_{m_{o} d_{i}}$ in fixed time. The joint position error of master-slave manipulators is shown in Fig.18. And the actual trajectory of underwater teleoperation systems is shown in Fig.19, which denotes the good tracking effect. In free motion, the master-slave position 

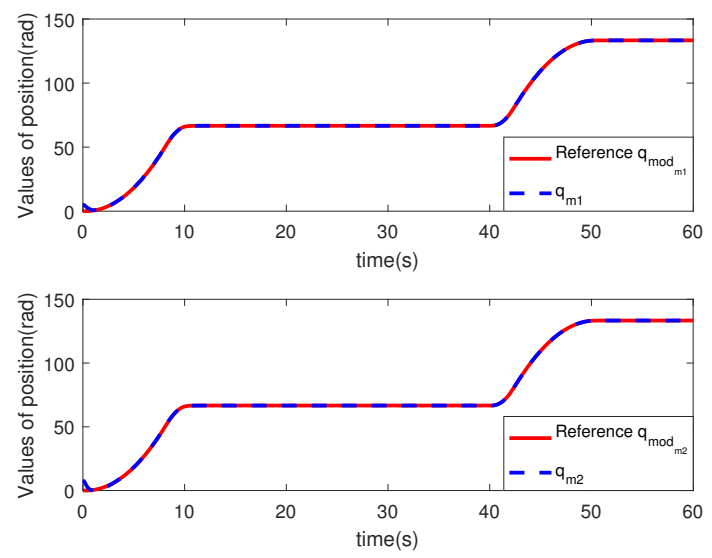

Figure 16: The values of reference impedance position and actual master position in free motion
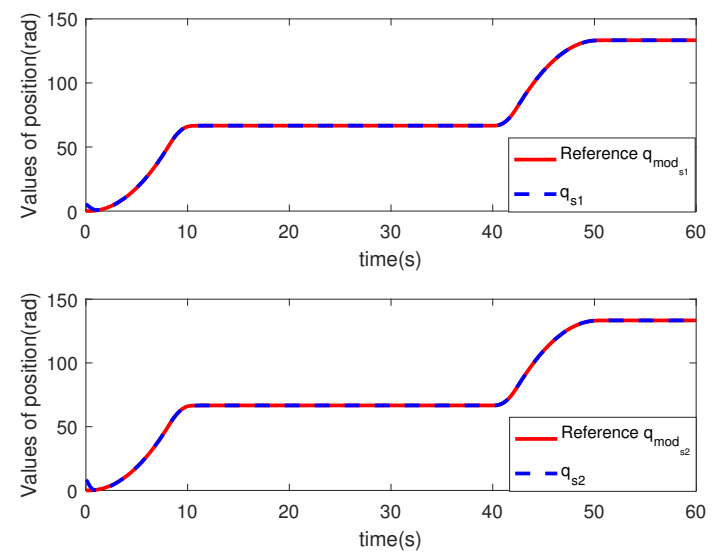

Figure 17: The values of reference impedance position and actual slave position in free motion
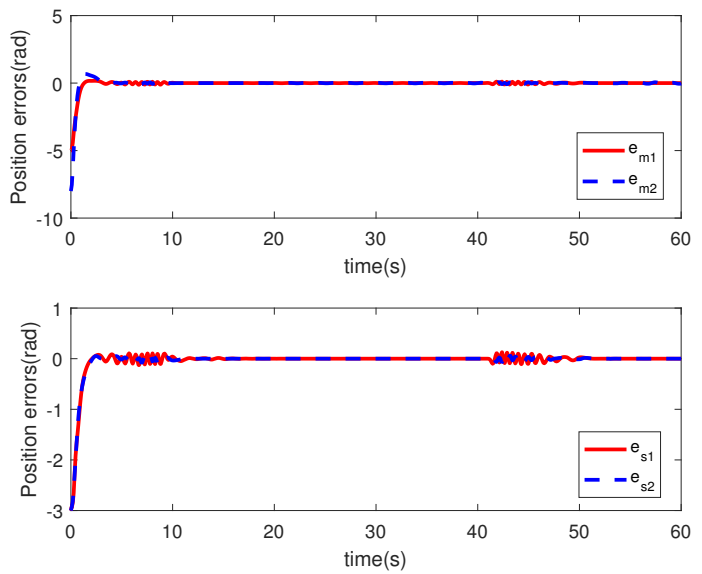

Figure 18: The values of position error for reference position and manipulators in free motion 

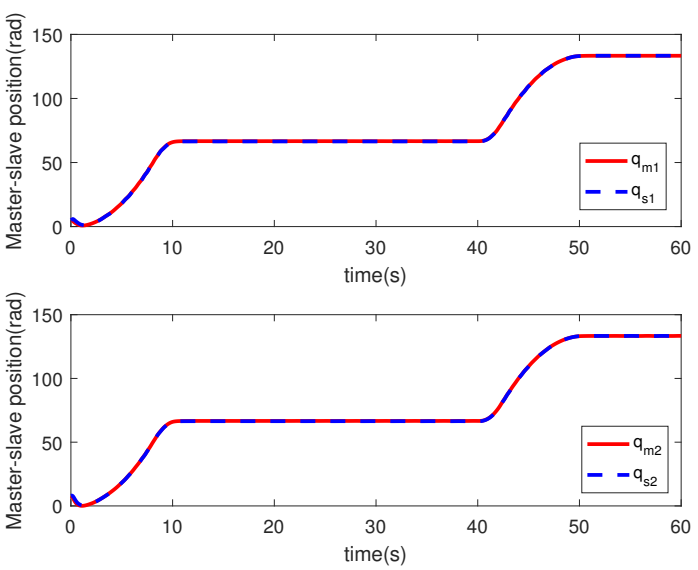

Figure 19: The values of position for master-slave manipulators in free motion

synchronization control is guaranteed.

For these simulation results, it is obvious that the fixed-time controller proposed in this paper gets good performance for system state convergence.

\section{Conclusion}

In this paper, a new fixed-time controller based on TDE is proposed for underwater teleoperation systems. To improve the transparency and flexibility of the master-slave manipulators, the impedance control models are presented to achieve force and position coordination control. A designed terminal sliding mode force observer is proposed to estimate the force without a sensor. In order to settle the problem of model uncertainties and external disturbances, a time delay estimator is proposed to approximate model uncertainties and external disturbances so that zero error model-free control is realized. With the fixed-time controller, the coordination control for master-slave manipulators is achieved with high speed, high precision, and strong robustness. The fixed settling time can be calculated using the formula with designed parameters. The stability analysis and simulation results are presented to illustrate the effectiveness of the proposed control method.

\section{Declarations}

\section{Data availability}

All data generated or analyzed during this study are included in this published article.

\section{Conflict of interest}

The authors declared that they have no conflicts of interest to this work. We declare that we do not have any commmercial or associative interest that represents a conflict of interest in connection with the work submitted.

\section{References}

[1] H. Joe, M. Kim, and S. cheol Yu, "Second-order sliding-mode controller for autonomous underwater vehicle in the presence of unknown disturbances," Nonlinear Dynamics, vol. 78, no. 1, pp. 183-196, 2014.

[2] E. Taha, Z. Mohamed, and K. Youcef-Toumi, "Trajectory tracking sliding mode control of underactuated auvs," Nonlinear Dynamics, vol. 84, no. 2, pp. 1079-1091, 2016. 
[3] N. Alexandros, K. V. Christos, and V. D. Dimos, "A tube-based mpc scheme for interaction control of underwater vehicle manipulator systems," IEEE/OES Autonomous Underwater Vehicle Workshop (AUV), pp. 1-6, 2018.

[4] J. Han, J. Park, and W. K. Chung, "Robust coordinated motion control of an underwater vehiclemanipulator system with minimizing restoring moments," Ocean Engineering, vol. 38, no. 10, pp. 11971206, 2011.

[5] S. Ganjefar, M. H. Sarajchi, and M. T. H. Beheshti, "Adaptive sliding mode controller design for nonlinear teleoperation systems using singular perturbation method," Nonlinear Dynamics, vol. 81, no. 3, pp. 1435$1452,2015$.

[6] L. Mohammadi, A. Alfi, and B. Xu, "Robust bilateral control for state convergence in uncertain teleoperation systems with time-varying delay: a guaranteed cost control design," Nonlinear Dynamics, vol. 88, no. 2, pp. 1413-1426, 2017.

[7] Y. Yang, C. Hua, J. Li, and X. Guan, "Fixed-time coordination control for bilateral telerobotics system with asymmetric time-varying delays," Journal of Intelligent and Robotic Systems, vol. 86, no. 3-4, pp. 447-466, 2017.

[8] T. Wang, Y. Li, J. Zhang, and Y. Zhang, "A novel bilateral impedance controls for underwater teleoperation systems," Applied Soft Computing, vol. 91, 2020.

[9] Y. Ji, D. Liu, and Y. Guo, "Adaptive neural network based position tracking control for dual-master/singleslave teleoperation system under communication check to constant time delays," ISA Transactions, vol. 93, pp. 80-92, 2019.

[10] F. Azimifar, M. Abrishamkar, B. Farzaneh, A. Sarhan, and H. Amini, "Improving teleoperation system performance in the presence of estimated external force," Robotics and Computer-Integrated Manufacturing, vol. 46, pp. 86-93, 2017.

[11] E. Slawiñski, V. Mut, and D. Santiago, "Pd-like controller for delayed bilateral teleoperation of wheeled robots," International Journal of Control, vol. 89, no. 8, pp. 1622-1631, 2016.

[12] K. Mohammadi, H. Talebi, and M. Zareinejad, "A novel position and force coordination approach in four channel nonlinear teleoperation," Computers and Electrical Engineering, vol. 56, pp. 688-699, 2016.

[13] G. Soheil, R. Sara, and H. Farzad, "Position and force tracking in nonlinear teleoperation systems with sandwich linearity in actuators and time-varying delay," Mechanical Systems and Signal Processing, vol. 86, pp. 308-324, 2017.

[14] M. Sharifi, H. Salarieh, and S. Behzadipour, "Impedance control of non-linear multi- dof teleoperation systems with time delay: absolute stability," IET Control Theory Applications, vol. 12, no. 12, pp. 1722 1729, 2018.

[15] J. Zhang, W. Liu, L. Gao, L. Li, and Z. Li, "The master adaptive impedance control and slave adaptive neural network control in underwater manipulator uncertainty teleoperation," Ocean Engineering, vol. 165, pp. $465-479,2018$.

[16] C. Hua, Y. Wang, Y. Yang, and X. Guan, "Force feedback control for bilateral teleoperation system with unknown prandtl-ishlinskii hysteresis," Journal of the Franklin Institute-Engineering and Applied Mathematics, vol. 357, no. 13, pp. 8321-8341, 2020. 
[17] A. M. D. Seyed, R. K. Hamid, S. Hamid, and E. Mohsen, "Observer-based adaptive force-position control for nonlinear bilateral teleoperation with time delay," Control Engineering Practice,, vol. 107, 2021.

[18] D. Zhai and Y. Xia, "Adaptive fuzzy control of multilateral asymmetric teleoperation for coordinated multiple mobile manipulators," IEEE Transactions on Fuzzy Systems, vol. 24, no. 1, pp. 57-70, 2016.

[19] R. Cui, C. Yang, Y. Li, and S. Sharma, "Adaptive neural network control of auvs with control input nonlinearities using reinforcement learning," IEEE Transactions on Systems, Man, and Cybernetics: Systems, vol. 47, no. 6, pp. 1019-1029, 2017.

[20] H. Koofigar, "Adaptive control of underwater vehicles with unknown model parameters and unstructured uncertainties," Proceedings of SICE Annual Conference (SICE), pp. 192-196, 2012.

[21] C. Hua, Y. Yang, and X. Guan, "Neural network-based adaptive position tracking control for bilateral teleoperation under constant time delay," Neurocomputing, vol. 113, pp. 204-212, 2013.

[22] Y. Wang, F. Yan, J. Chen, and F. Ju, "A new adaptive time-delay control scheme for cable-driven manipulators," IEEE Transactions on Industrial Informatics, vol. 15, no. 6, pp. 3469-3481, 2019.

[23] J. Kim, H. Joe, S. Yu, J. S. Lee, and M. Kim, "Time-delay controller design for position control of autonomous underwater vehicle under disturbances," IEEE Transactions on Industrial Electronics, vol. 63, no. 2, pp. 1052-1061, 2016.

[24] J. Lee, P. Chang, and M. Jin, "Adaptive integral sliding mode control with time-delay estimation for robot manipulators," IEEE Transactions on Industrial Electronics, vol. 64, no. 8, pp. 6796-6804, 2017.

[25] Y. Wang, K. Zhu, F. Yan, and B. Chen, "Adaptive super-twisting nonsingular fast terminal sliding mode control for cable-driven manipulators using time-delay estimation," Advances in Engineering Software, vol. 128, pp. 113-124, 2019.

[26] D. Lee and M. Spong, "Passive bilateral teleoperation with constant time delay," IEEE Transactions on Robotics, vol. 22, no. 2, pp. 269-281, 2006.

[27] C. Hua and X. Liu, "Teleoperation over the internet with/without velocity signal," IEEE Transcations on Instrumentation and Measurement, vol. 60, no. 1, pp. 4-13, 2011.

[28] H. Zhang, A. Song, H. Li, and S. Shen, "Novel adaptive finite-time control of teleoperation system with time-varying delays and input saturation," IEEE Transactions on Cybernetics, vol. 51, no. 7, pp. 3724-3737, 2021.

[29] M. Kim, D. Kim, and K. Park, "Sliding mode controller with piecewise linear sliding surface for secondorder nonlinear systems," SICE-ICASE International Joint Conference, vol. 1-13, pp. 4519-+, 2006.

[30] Y. Yang, C. Hua, and X. Guan, "Adaptive fuzzy finite-time coordination control for networked nonlinear bilateral teleoperation system," IEEE Transactions on Fuzzy Systems, vol. 22, no. 3, pp. 631-641, 2014.

[31] L. Wang, Z. Song, X. Liu, and Z. Li, "Continuous finite-time integral sliding mode control for attitude stabilization," IEEE Transactions on Circuits and Systems II: Express Briefs, vol. 67, no. 10, pp. 2084$2088,2020$.

[32] H. Zhang, A. Song, H. Li, D. Chen, and L. Fan, "Adaptive finite-time control scheme for teleoperation with time-varying delay and uncertainties," IEEE Transactions on Systems, Man, and Cybernetics: Systems, vol. 52, no. 3, pp. 1552-1566, 2022. 
[33] S. S.-D. Xu, C.-C. Chen, and Z.-L. Wu, "Study of nonsingular fast terminal sliding-mode fault-tolerant control," IEEE Transactions on Industrial Electronics, vol. 62, no. 6, pp. 3906-3913, 2015.

[34] H. Sai, Z. Xu, S. He, E. Zhang, and L. Zhu, "Adaptive nonsingular fixed-time sliding mode control for uncertain robotic manipulators under actuator saturation," ISA Transactions, 2021.

[35] N. Hogan, "Impedance control: an approach to manipulation: part 1 - theory," Journal of Dynamic Systems, Measurement, and Control, vol. 107, no. 1, pp. 1-7, 1985.

[36] S. Bhat and D. Bernstein, "Finite-time stability of continuous autonomous systems," SIAM Journal on Control and Optimization, vol. 38, no. 3, pp. 751-766, 2000.

[37] A. Polyakov, "Nonlinear feedback design for fixed-time stabilization of linear control systems," IEEE Transactions on Automatic Control, vol. 57, no. 8, pp. 2106-2110, 2012.

[38] Z. Zuo, "Nonsingular fixed-time consensus tracking for second-order multi-agent networks," Automatica, vol. 54, pp. 305-309, 2015.

[39] S. Jung, T. Hsia, and R. Bonitz, "Force tracking impedance control of robot manipulators under unknown environment," IEEE Transactions on Control Systems Technology, vol. 12, no. 3, pp. 474-483, 2004.

[40] M. Jin, J. Lee, P. Chang, and C. Choi, "Practical nonsingular terminal sliding-mode control of robot manipulators for high-accuracy tracking control," IEEE Transactions on Industrial Electronics, vol. 56, no. 9, pp. 3593-3601, 2009. 